\title{
Characterization and Molecular Identification of Poly Urethane Degrading Bacteria
}

\author{
V. Shree Yazhini (D), M. Lakshmi Prabha* (D) and Reya Issac \\ Department of Biotechnology, Karunya Institute of Technology and Sciences, \\ Coimbatore - 641 114, Tamil Nadu, India.
}

\begin{abstract}
Plastics can be considered as the world's largest pollution that is accumulating in the environment. The degradation of these plastics is difficult because they are accumulated over years and also they are dumped in an area which causes more environmental problems. To reduce the environmental pollution under the waste management with the help of microorganism was taken into account. In those bacteria plays a major role in controlling those problems. Polyurethane can be considered as one of the most used synthetic polymers in the world. Hence, degradation of polyurethane was taken into account in the study with the help of bacteria. In this experiment, we isolated and identified the bacteria that can degrade the polyurethane from the polyurethane buried soil sample. The isolated organism was sequenced and identified at the species level. Enzyme activities of those organisms were also identified along with the degradation process. With the help of this study, we identified a novel bacterium that can degrade polyurethane.

Keywords: Degradation, Poly Urethane, Identification, Biodegradation, 16s rRNA Sequencing
\end{abstract}

(C) The Author(s) 2021. Open Access. This article is distributed under the terms of the Creative Commons Attribution 4.0 International License which permits unrestricted use, sharing, distribution, and reproduction in any medium, provided you give appropriate credit to the original author(s) and the source, provide a link to the Creative Commons license, and indicate if changes were made. 


\section{INTRODUCTION}

Plastics are used widely by people in various forms and structures around the world. Plastics are introduced for convenience of people to improve the quality of life. Because of the increasing quantity of plastic waste and their effects and safe disposal of those plastics has become a public concern. The degradation of plastic waste will take a very long time and also it causes natural concerns about the pollution. Because of the contamination caused by dumping of the plastic wastes, some reports have confirmed that some plastic products can mimic the human hormones and cause the human health hazards. Dumping of plastic waste was also a concern on the marine ecosystem pollution and causes major crises to most of the living organisms in the sea as well as land.

Polyurethanes are formed with the links between urethane units. It is a polymer which is formed by a reaction between a polyol with di-isocyanate or a polymeric isocyanate. These polymers are used in the buildings and construction automotive industry electronics medical packaging industry architecture and marine industry etc. These can be made into fine threads used in garments, rigid forms for freezer insulation system, car seats, bumpers, car body, encapsulate, microelectronic components, underwater cables, cushioning materials, catheter and tubing, surgical drapes, wound dressing, packaging for shipping etc. The types of polyurethane are thermoplastics, flexible foams, rigid foams, binders, reaction injection moulding, and waterborne polyurethane dispersions. Depending on the manufacturing process polyurethanes can be called as unique because it is manufactured by three-step process that are production of isocyanates, production of polyols and production of polyurethanes because the both isocyanates and polyols are liquids which combined to form polyurethanes polymer ${ }^{1}$.

The properties of the following PU depends upon both the method of the preparation and what type of monomer is used. Polyurethane is synthesized between the alcohols and isocyanates in the exothermic reaction. Polyurethanes can be classified in polyester polyurethanes or polyether polyurethanes. That depends upon the substrate that is used and the biological attack towards them can be determined by the type of substrate for the synthesis ${ }^{2}$. The polyester polyurethanes that contain repeated ester bond linkage that can be degraded under various chemical and biological conditions because of the cleavage of ester bonds ${ }^{3}$. From various literatures we can identify that there is a wide group of microorganisms such as bacteria and fungi were found to degrade the polyurethane through the use of some enzymes such as esterase, amidase, urease and protease $\mathrm{A}^{4}$.

There are various methods that can be used for degradation of polyurethane such as temperature, radiation and also microbes that can be considered as biodegradation. But the degradation using temperature has provided only about $50 \%$ of degradation and it has also produced toxic substances such as Oil, gas, ash, cyanide, isocyanates, ammonia, nitrogen oxides at the temperature of 330 to $800^{5}$. Hence the use of temperature for degradation is very harmful and costly. so the usage of microorganisms for degradation will be cost effective and also ecofriendly.

In this study we are concentrating on the biological degradation of PU. The microbes can be used for degradation of polyurethane there are various mechanisms that identified for the degradation of polyurethane by biological organisms such as bacteria or fungi.

Due to the increase in the usage of plastics the disposing of those materials were becoming become very hard. It is known that there are millions and millions tons of plastic dumped in oceans and lands annually. This dumping has many environmental concerns such as pollutions, biodiversity reduction etc. They affect the living organisms in both marine and land. Hence in this research we took common commodity plastic polyurethane and the bacteria that can degrade PU was identified. In this experiment the bacteria is isolated and characterized in very detailed manner and the degradation of the polyurethane sheets are also determined and analysed.

\section{MATERIALS AND METHODS Materials}

PU sheets, Nutrient agar and Nutrient broth were purchased from Sigma-Aldrich. mineral salt medium 6 was prepared as Following Grams per liter, $\mathrm{K}_{2} \mathrm{HPO}_{4}-0.10, \mathrm{KH}_{2} \mathrm{PO}_{4}-0.06$, $\mathrm{NaCl}-0.6, \mathrm{CaCl}_{2} .2 \mathrm{H}_{2} \mathrm{O}-0.005,\left(\mathrm{NH}_{4}\right)_{2} \mathrm{SO}_{4}-0.8$, 
$\mathrm{MgSO}_{4} \cdot 7 \mathrm{H}_{2} \mathrm{O}-0.05, \mathrm{FeSO}_{4}-0.004$, AGAR AGAR-20g. without the carbon source as degradation media and without agar agar for liquid media. The gram staining kit was also purchase from hi-media. The test kits for IMViC test was also purchased and used. The skim milk powder and smashed potato-10g, dextrose-1g, agar powder- $0.875 \mathrm{~g}$ and distilled water of $50 \mathrm{ml}$ are used for protease and amylase test respectively.

Note: All the media, chemicals or reagents required for the experiments were dissolved or prepared by using distilled or deionized water. And all glassware were washed by using detergents and which was air dried. The dried glassware was again sterilized by using autoclave at $121^{\circ} \mathrm{C}$ for $15 \mathrm{~min}$. Sample Collection

The $5 * 5 \mathrm{~cm}$ polyurethane sheets are buried in the soil for over six months. The soil around that polyurethane sheet is collected. The collected soil sample was used for isolation of the microorganisms

\section{Isolation Of Micro Organism Serial Dilution}

The microorganisms are enumerated from the soil by serial dilution method. In this method $1 \mathrm{~g}$ of collected soil is mixed with $10 \mathrm{ml}$ of distilled water which can be considered as a stock solution. Then 8 Eppendorf's taken and are labelled as S1,S2,S3,S4,S5,S6,S7,S8. In S1 $100 \mu \mathrm{l}$ of your working solution is taken and $900 \mu \mathrm{l}$ of distilled water is added. Then in $\mathrm{S} 2500 \mu$ solution from $\mathrm{S} 1$ and $500 \mu \mathrm{l}$ of distilled water is added. The same procedure is carried out by adding $500 \mathrm{ml}$ of solution from the previous Eppendorf to the current Eppendorf in a serial manner till sample is diluted to $10^{8}$.

\section{Spread Plate Method}

Sample from S6 and S8 are used for isolating the organism through spread plate method. In the spread plate method $100 \mu \mathrm{l}$ of sample is taken and spread with the help of $\mathrm{L}$ rod on the solidified nutrient agar medium. The nutrient agar medium is prepared by addition of $3 \mathrm{~g}$ of nutrient agar powder to $1000 \mathrm{ml}$ of distilled water and autoclaved. The medium is incubated for 24 hours at $37^{\circ} \mathrm{C}$ and the CFU value is calculated for the two plates.

\section{Total Heterotrophic Count}

The CFU value is calculated with the help of normal counting in the naked eye and the results are in the following. In this method the plates are divided into 4 parts and the numbers of colonies are calculated by marking.

C.F.U. /g= Number of colonies/ inoculum size $(\mathrm{ml}) \mathrm{X}$ dilution factor

\section{Colony Selection}

Then more than 200 different colonies are selected and grown in the agar plate. But for the isolation of plastic degrading bacteria a special mineral salt medium ${ }^{6}$ was prepared as Following Grams per liter, $\mathrm{K}_{2} \mathrm{HPO}_{4}-0.10, \mathrm{KH}_{2} \mathrm{PO}_{4}-0.06$, $\mathrm{NaCl}-0.6, \mathrm{CaCl}_{2} .2 \mathrm{H}_{2} \mathrm{O}-0.005,\left(\mathrm{NH}_{4}\right)_{2} \mathrm{SO}_{4}-0.8$, $\mathrm{MgSO}_{4} \bullet 7 \mathrm{H}_{2} \mathrm{O}-0.05, \mathrm{FeSO}_{4}-0.004$, AGAR AGAR-20g. Then all the chemicals are added in one litre of distilled water and thus autoclave and poured in the Petri plate in the sterile condition is taken and in those two organisms (T1 and $\mathrm{T} 2$ ) were grown successfully. In this study the polyurethane is used as a carbon source for organism identification.

\section{Identification Of Micro Organism}

The bacteria is identified by its physical properties such as the size, shape, margin, texture, elevation, opacity and pigment along with gram staining for structural properties such as the shape, size and arrangement. Also by the biochemical tests such as IMViC test, catalase test and the motility test by hanging drop method.

\section{Growth Kinetics}

Microbial growth kinetics can be defined as the relationship between the specific growth rates of the microbial population along with the substrate concentration. The bacterial culture was inoculated in a $50 \mathrm{ml}$ of a nutrient broth which is marked $\mathrm{T} 1$, and then the optical density was taken at $620 \mathrm{~nm}$ for all the three bacterial cultures along with control. Then the optical density is taken after 9 days to the death phase of the organisms and the graph is plotted.

\section{Enzyme Analysis}

\section{Protease Test}

Protease is considered as a group of enzymes and its main characteristic function is hydrolysed the peptide bonds of the protein and break them down into polypeptide chains are free amino acids. There are various types of enzymes which help in the degradation of polyurethane and protease is one of the enzymes that can degrade the polyurethane. Using slim milk agar can be considered as one of the methods that can be used to check if the bacteria will produce protease 
enzyme or not. The skim milk agar is prepared by adding $5 \mathrm{~g}$ of skim milk powder and $1 \mathrm{~g}$ of nutrient agar in $1 \mathrm{ml}$ distilled water and autoclaved. The protease is an intracellular or extracellular enzyme that is produced by the organism and can be identified by using this method. In this method after the addition of organism and incubation the formation of a clear zone around the place where the organism is placed can be considered as the presence of protease enzyme in the organism.

The procedure that is used in this study is as follows: Skim milk Agar is prepared and 4 wells are created in which 2 wells contain 2 pellets and 2 supernatants with the triton $X-100$ of the three different organisms are used. Then in those wells three pellet solutions with detergent and three supernatant and solution with detergent are added to different organisms respectively. Then the organisms are incubated for over 24 hours and the formation of a clear zone is observed.

\section{Amylase Test}

The main function of amylase enzyme is to hydrolyse the glycosidic bonds in starch molecules by converting the complex carbohydrates into simple sugars. This enzyme is used to reduce the molecular weight of the polymer during the gradation process. There are various types of enzymes which help in the degradation of polyurethane and amylase is one of the enzymes that can degrade the polyurethane. Using Potato dextrose Agar can be considered as one of the methods that can be used to check if the bacteria will produce amylase enzyme or not. The same procedure as protease test was followed. The PDA agar was prepared by addition of smashed potato- $10 \mathrm{~g}$, dextrose- $1 \mathrm{~g}$, agar powder- $0.875 \mathrm{~g}$ and distilled water of $50 \mathrm{ml}$ and autoclaved.

\section{Degradation Of Poly Urethane}

The PU sheets are cut into $5 \mathrm{~cm} * 5 \mathrm{~cm}$ and the initial weight of the sheets is taken. Then the sheets are placed on mineral salt agar and mineral salt broth with each sheet in each organism respectively. Then the organisms along with PU sheets are incubated for 45 days. Then the sheets are taken and the final weight is measured. The organism which has fast degradation activity is taken for SEM analysis. The quick degradation is identified by measuring the final weight of the sheets.

The sheet which weight is decreased is taken for analysis.

\section{Molecular Identification}

The molecular identification is essential steps for the identification of bacteria in species level. The molecular techniques are considered as a modern tool for the identification and classification of bacteria. The most common morphological method used for the identification of molecular characteristics of a Bacterium is 16s rRNA gene sequencing method.

The $16 s$ rRNA sequencing is carried out by genomic DNA isolation, PCR amplification, sequencing and applying bioinformatics. Genomic DNA is isolated by homogenization by lysis buffer then binding by binding buffer then washing and elution, the DNA concentrations were measured by $1 \%$ agarose gel electrophoresis. The PCR amplification as carried out with the help of Taq DNA polymerase is supplied in $2 X$ Taq buffer, $0.4 \mathrm{mM}$ dNTPs, $3.2 \mathrm{mM} \mathrm{MgCl} 2$ and $0.02 \%$

Table 1. The size, shape, margin, texture, elevation, opacity, pigment of two organisms are noted

\begin{tabular}{lccccccc}
\hline Organism & Size & Shape & Margin & Texture & Elevation & Opacity & Pigment \\
\hline T1 & Big & Circular & Entire & Smooth & Flat & Opaque & Nil \\
T2 & Small & Puncta form & Fimbriate & Smooth & Raised & Opaque & Nil \\
\hline
\end{tabular}

Table 2. The shape, size, arrangement and motility of the two organisms are noted

\begin{tabular}{|c|c|c|c|c|c|}
\hline Organism & Gram Reaction & Shape & Size & Arrangement & Motility \\
\hline $\mathrm{T} 1$ & Gram positive & Rod & Long & Single or pair & Non-motile \\
\hline $\mathrm{T} 2$ & Gram positive & Rod & Small & $\begin{array}{l}\text { Single, pair or } \\
\text { in short chain }\end{array}$ & Motile \\
\hline
\end{tabular}


bromophenol blue along with universal primer 27F AND 1492R. The main stages of PCR as carried out for 25 cycles and the product was purified. The single pass sequencing was carried out on each primer with 16s rRNA universal primers. In bioinformatics the obtained sequence was analysed for its similarity using NCBI blast similarity search tool. Then the phylogeny analysis was performed following by the multiple sequence alignment using the program MUSCLE $3.7^{7}$, The resulting aligned sequences were cured using the program Gblocks 0.91b.This Gblocks eliminates poorly aligned positions and divergent regions (removes alignmentnoise) ${ }^{8}$. Finally, the program PhyML 3.0 aLRT was usedfor phylogeny analysis and HKY85 as Substitution model. The program Tree Dyn 198.3 was used for tree rendering ${ }^{9}$.

\section{Surface Analysis}

Sem Analysis

Scanning electron microscopy is an instrument which helps in the detection of surface modification of polymers during degradation. In these methods the topological changes after polymer surfaces such as formation of holes and

Table 3. Summary of the biochemical tests such as IMVIC and catalase test results are shown

\begin{tabular}{lll}
\hline Organism & T1 & T2 \\
\hline Indole Production & - & - \\
Methyl red & + & - \\
Voges-Prauskauer (VP) & - & - \\
Citrate utilization & - & - \\
Catalase & + & + \\
\hline
\end{tabular}

The biochemical test is given in which + is positive and - is negative.

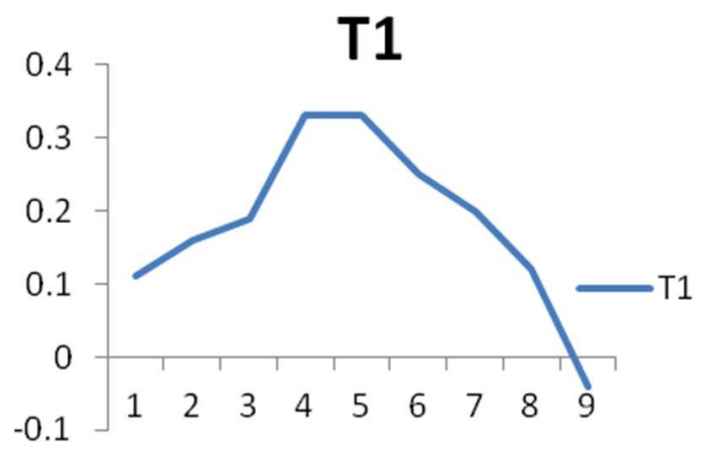

cracks, roughness increase or the attachment of the microbes to the surfaces of the plastics can be imaged. Typical changes in the polymer morphology visible such as cracks and cavities, surface degradation and deterioration of the polymer films can also be seen in the help of SEM.

\section{Experimental Results}

Total Heterotrophic Count

In this study the colony forming unit (C.F.U.) of the S6 and S8 of the samples are calculated and the results are as follows:

C.F.U. $/ g=$ Number of colonies/inoculum size $(\mathrm{ml}) \mathrm{X}$ dilution factor

For S6 $=3225 / 1 * 10^{-6}$

C.F.U. $/ g$ of $S 6=322.5^{*} 10^{-5}$

For S $8=1816 / 1 * 10^{-8}$

C.F.U. $/ g$ of $S 8=181.6 * 10^{-7}$

\section{Identification Of Micro Organism}

Colony Morphology

The colony morphology of the bacteria as clearly identified and noted in the Table 1.

\section{Gram Staining And Motility}

The grams reaction, size, shape and arrangement of the two organisms along with the motility test by hanging drop method as noted in Table 2.

\section{Biochemical Tests}

The summary of the results obtained by the biochemical tests such as indole, methyl red Voges-Prauskauer, citrate utilization and catalase test are given in the Table 3.

\section{Growth Kinetics}

The growth curves of the two organisms are calculated till the death phase, the optical density is $620 \mathrm{~nm}$ were given in the "Fig. 1." and "Fig. 2."

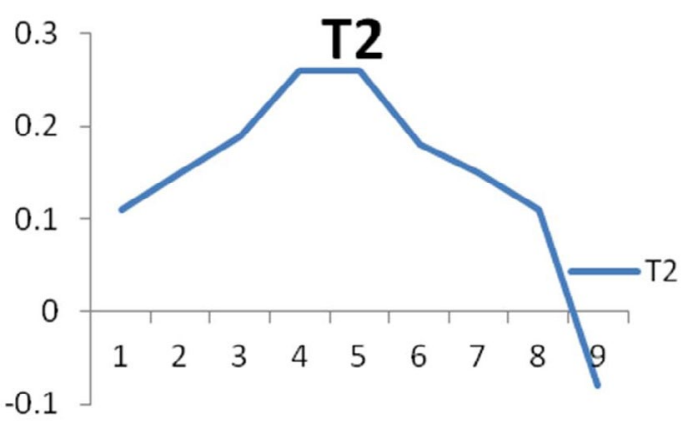

Fig. 1. Graph represents the growth curve of organism T1

Fig. 2. Graph represents the growth curve of organism T2 


\section{Enzymes Analysis}

The enzymes are analysed and the presence of protease and amylase enzymes are analysed. The protease enzyme in both organisms shows positive results while the amylase shows negative results.

\section{Biodegradation of Polyurethane}

In this experiment the PU sheets were kept in both solid and liquid medium. After 45 days the weight is analysis. Then Bacillus licheniformis SY2 is taken for analysis. The solid medium ("Fig.4." for T1 and "Fig. 5." for T2) and liquid medium ("Fig.3.") with the sheets are shown.

\section{Molecular Identification}

The molecular identification for fast degrading bacteria as made by $16 \mathrm{~s}$ rRNA sequencing and the results obtained in the name of SY2 for T2. The obtained results are as follows Partial 165 gene of 475 bp was obtained after sequencing and shown below in FASTA format.

SY2

CONTIG_SY_2

GCTCCCTTAGGTCA GCG G CG GACG GTGA GTAACACGTGGGTAACCTGCCTGTAAGACTGGGA TAACTCCGGGAAACCGGGGCTAATACCGG

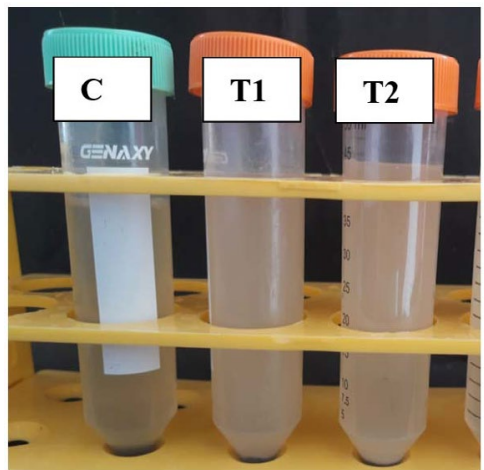

Fig. 3. The PU sheets are immersed in liquid media

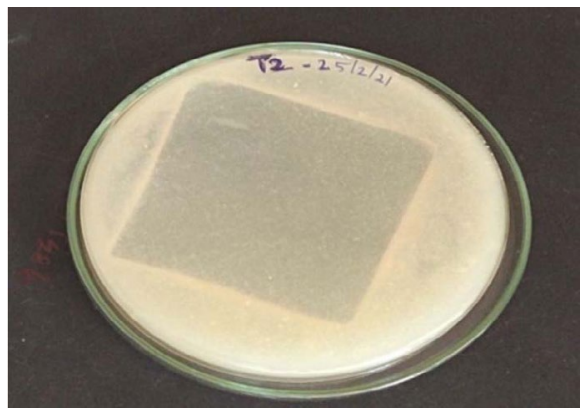

Fig. 5. PU sheet in solid medium with $\mathrm{T} 2$.
ATGCTTGATTGAACCGCATGGTTCAATCATAA AAGGTGGCTTTTAGCTACCACTTACAGAT GGACCC GCG G C CATTAGCTAGTTGGTGAGGTAAC GGCTCACCAAGGCGACGATGCGTAGCCGACC TGAGAGGGTGATCGGCCACACTGGGACTGAGA CACGGCCCAGACTCCTACGGGAGGCAGCAGTAGGG AATCTTCCGCAATGGACGAAAGTCTGACGGAGCAA CGCCGCGTGAGTGATGAAGGTTTTCGGATCGTAAAA CTCTGTTGTTAGGGAAGAACAAGTACCGTTCGAATA GGGCGGCACCTTGACGGTACCTAACCAGAAAGCCAC GGCTAACTACGTGCCAGCAGCCGCGGTAATACGTA GGTGGCAAGCGTTGTCCGGAATTATTGGGCGTAAAG CGCGCGCAGGCGGTTTCTTAAGTCTGATGTGAAA GCCCCCGGCTCAACCGGGGAGGGTCATTGGAAACT GGGGAACTTGAGTGCAGAAGAGGAGAGT GGAATTCCACGTGTAGCGGTGAAATGCGTAGAGATG TGGAGGAACACCAGTGGCGAAGGCGACTCTCTGG TCTGCAACTGACGCTGAGGCGCGAAAGCGTGGGGA GC GAACAGGATTAGATACCCTGGTAGTCCACGCCGT AAACGATGAGTGCTAAGTGTTAGAGGGTTTCCGCCC TTTAGTGCTGCAGCAAACGCATTAAGCACTCCGCCT GGGGAGTACGGTCGCAAGACTGAAACTCAAAGGAA TTGACGGGGGCCCGCACAAGCGGTGGAGCATGTGG TTTAATTCGAAGCAACGCGAAGAACCTTACCAGGTCT TGACATCCTCTGACAACCCTAGAGATAGGGCTT

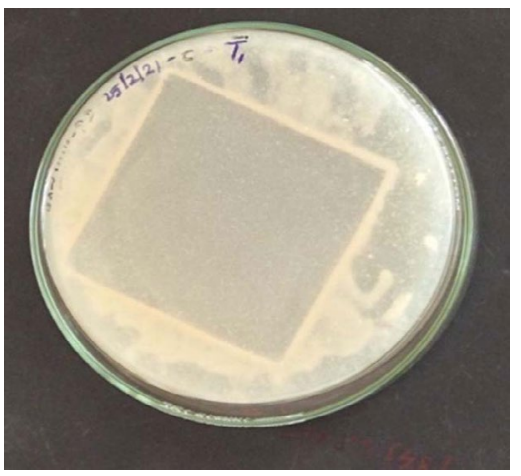

Fig. 4. PU sheet in solid medium with T1.

CCCCTTCGGGGGCAGAGTGACAGGTGGTGCATGGTT GTCGTCAGCTCGTGTCGTGAGATGTTGGGTTAAGT CCCGCAACGAGCGCAACCCTTGATCTTAGTTGCCAGC ATTCAGTTGGGCACTCTAAGGTGACTGCCGGT GACAAACCGGAGGAAGGTGGGGATGACGTCAAATC ATCATGCCCCTTATGACCTGGGCTACACACGTGC TACAATGGGCAGAACAAAGGGCAGCGAAGCCGCGA GGCTAAGCCAATCCCACAAATCTGTTCTCAGTT CGGATCGCAGTCTGCAACTCGACTGCGTGAAGCTGG 


\section{AATCGCTAGTAATCGCGGATCAGCATGCCGCGGT GAATACGTTCCCGGGCCTTGTACACACCGCCCGTCAC ACCACGAGAGTTTGTAACACCCGAAGTCGGTGA GGTAACCTTTTGGAGCCAGCCGCCGAAGGTGGG}

\section{RESULT}

\section{Bacillus licheniformis}

BLAST result showed that the test organism (SY2) was similar to Bacillus licheniformis with $100 \%$ similarity and $E$ value 0.0 .

Query ID - Icl|Query_3355

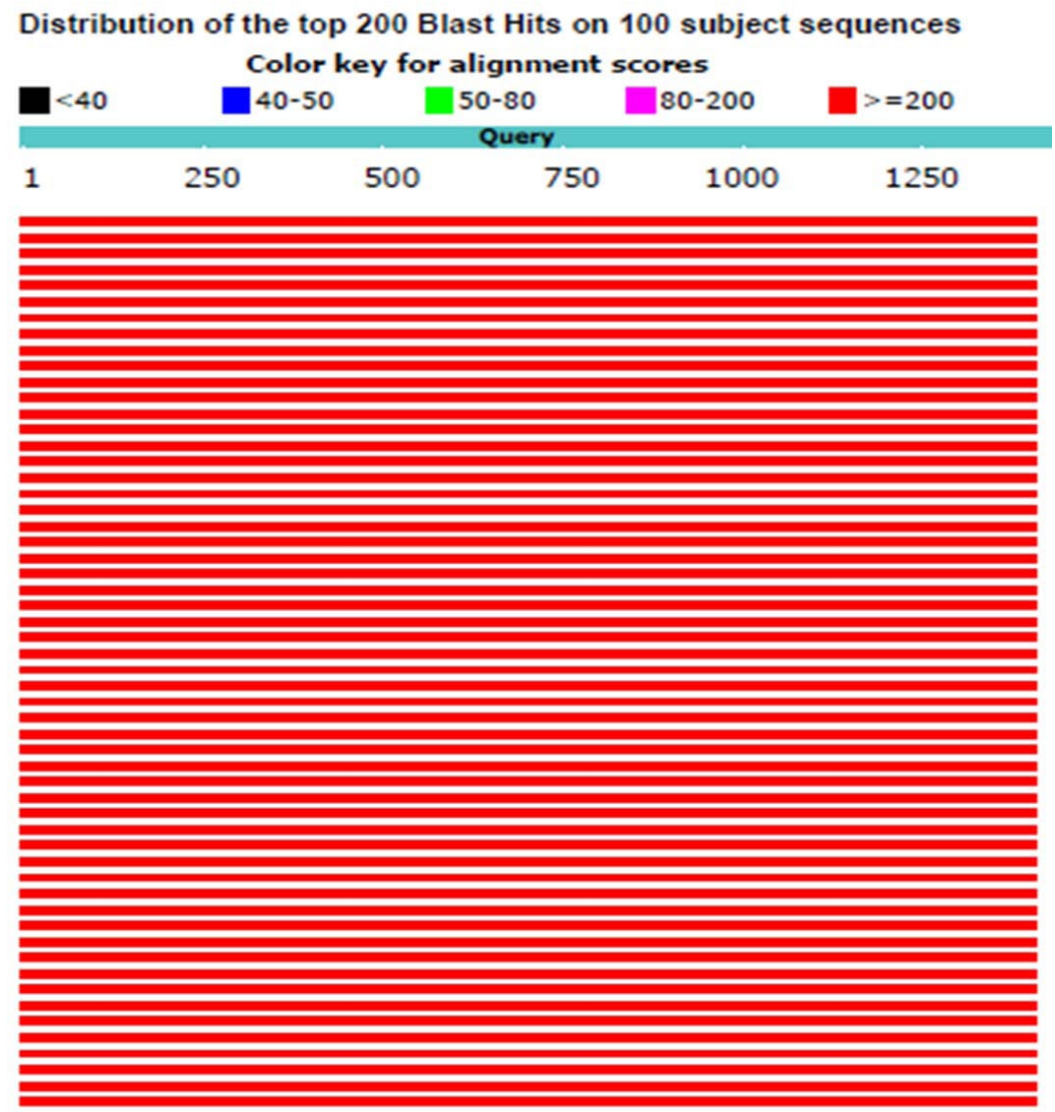

Fig. 6. Alignment of the BLAST query for SY2

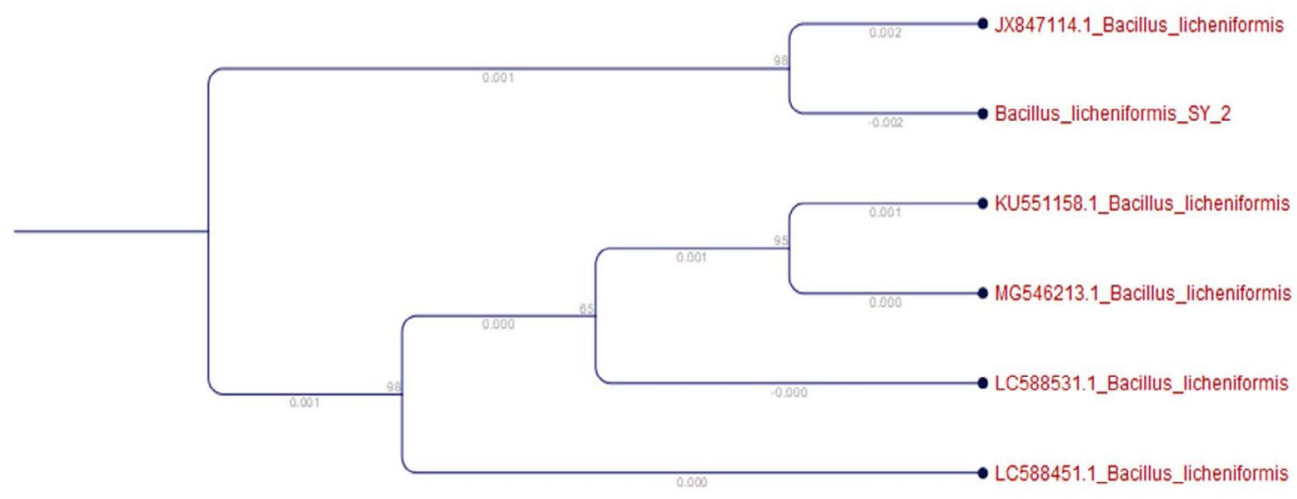

Fig. 7. the phylogenetic tree of SY2 
Description - CONTIG_SY_2

Molecule type - dna

Query Length - 1400

Graphic Summary

The distribution on the 100 sequences with help of top 200 Blast hits are shown in "Fig.6." Phylogeny Analysis

The phylogeny of the obtained microorganism is identified and the phylogenetic tree is obtained and given below. The phulogenetic tree of SY2 (Bacillus licheniformis) as given in the "Fig.7."

\section{NCBI Submission}

The DNA of the organism is submitted to NCBI - GENBANK and the accession number is obtained as SY2, Organism - Bacillus licheniformis, Accession number - MW857275.

SEM Analysis

In the SEM analysis we can see some corrosion and holes in the PU Sheet. the sheet that was acted by Bacillus licheniformis SY2 is taken and the analysed results are provided in "Fig. 8." These abnormalities in the sheet prove that the microorganism is degrading the sheet. The specific bacteria has the ability to degrade faster can be seen.

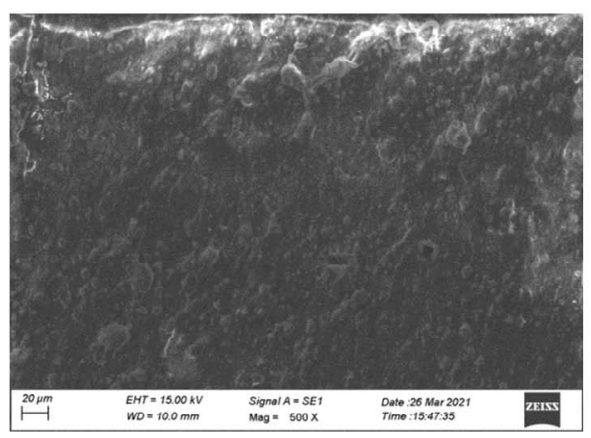

\section{DISCUSSION}

There were investigations that were carried out for identification of PU degradation is carried out and the samples that are collected were buried in soil collected from plastic waste disposal sites ${ }^{6}$. In our study the PU sheets were buried in normal farming soil for over 6 months. So the study can say that PU degrading bacteria are present in normal soil also.

In an experimental study we identified that the certain mineral salt medium has the ability to grow polyurethane degrading bacteria in it by using polyurethane as an only source of carbon and nitrogen ${ }^{6}$. The organisms are isolated from the soil where the polyurethane sheets are buried for over 6 months and the surrounding bacteria's are taken so it can be considered that these organisms can have the activity to degrade polyurethane. And these organisms were grown in the special minerals of media that can degrade polyurethane. The biochemical test of this entire organism was conducted and the morphology and structural characteristics of this organism are identified.

The growth curve of the organisms were also interpreted so that the growth of the organism can be identified and it may help in the analysis of
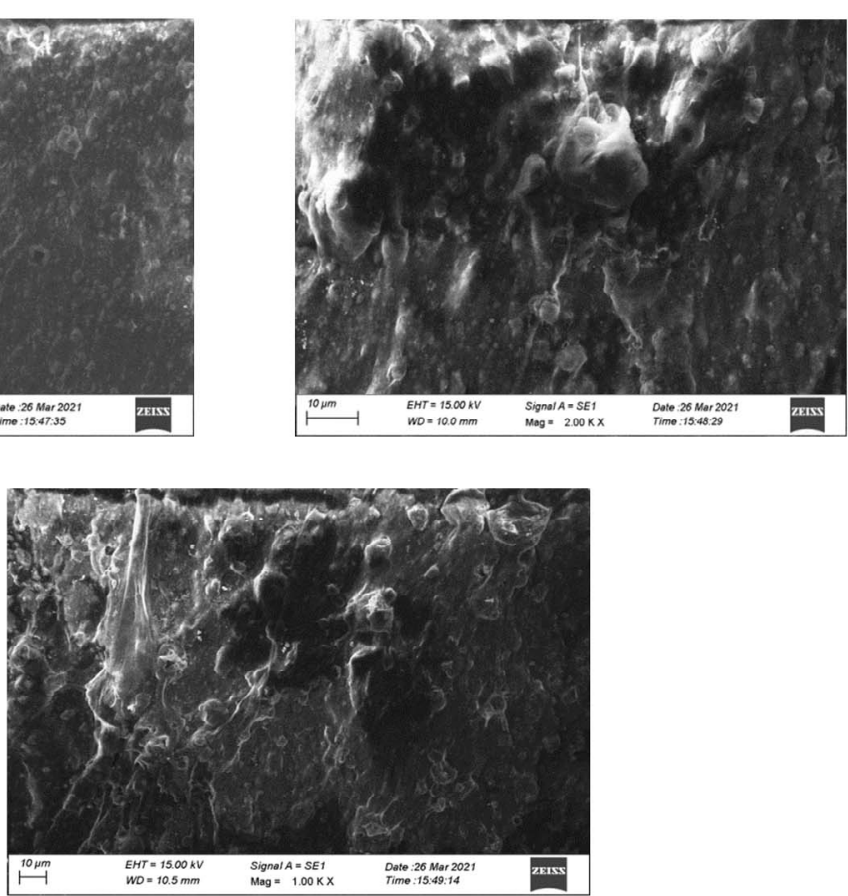

Fig. 8. Shows the surface degradation of the PU sheet in SEM 
the production of enzymes during its growth and also the time it takes for the complete growth and death of the organism.

In this study there is no any leaching of plastics in the degradation medium because medium only contains mineral salts and agar-agar (only used for solidification). So there is no chance of leaching substance in medium alone. After the growth of micro-organism in the medium we can see the effects on the surface of the PU sheets. Also the control in the experiment has no any changes in the PU sheet surface that was it has same weight before and after placing in medium in control. So we can conclude that only microorganism was responsible for degradation.

The molecular identification of the organism was conducted and the organisms are identified in a species level which will be very helpful in conducting the experiment for degradation of polyurethane. The phylogenetic tree was derived for the organism and submitted to NCBI GENBANK and an accession number for organism was obtained which is a mandatory process in isolation and identification of any microbes.

In a study the scientists identified the organisms capable of degrading polyester polyurethane such as Pseudomonas chlororaphis, Bacillus subtilis, Comamonas acidovorans, and Acinetobacter gerneril. They also identified the enzymes such as lipase and esterase that can degrade the Polyester polyurethane ${ }^{10}$. While in our study we used pure polyurethane and identified its degradation and the presence of enzymes in the intracellular or extracellular surface of the organism and its effect in the degradation process are observed. The organism we obtained was Bacillus licheniformis and the enzyme activity in that organism that can be used for catalysing the degradation process was protease. The protease enzyme produced was intracellular enzyme.

There are various researches says that the PU biodegradation was occurring with the help of bacteria or fungi. But most of the research will stop at some point of view but the PU degradation that was made by study can be considered as one of the research that was carried out in the way to tell us that the PU degrading bacteria isolation to the identification and the level of degradation checking ${ }^{5}$. In this study we can identify the organism that can degrade the PU sheets most effectively than those of other isolated organism. This was observed by keeping the polyurethane sheets in the solid and liquid media and the initial weight of the sheet was taken after certain period of time the final weight was checked and from that we can identify that certain bacteria called Bacillus licheniformis SY2 has the ability to degrade the polyurethane sheets in a fastest speed than compared to that of other organism. The sheets that are degraded by Bacillus licheniformis SY 2 has was taken for surface analysis and the corrosive nature of the organism on the polyurethane sheets and the holes appeared in the sheets has proven that the this organism is degrading the polyurethane sheets and it is degradation positive organism.

\section{CONCLUSION}

From this experiment we can conclude that the PU degrading bacteria can be isolated from soil. And the enzyme protease can also help in catalysing the biodegradation process along with bacteria. This study also shows that the PU sheets are buried in soil for 6 months and the degrading bacteria's were isolated. They the degradation activity of the isolated bacteria Bacillus licheniformis has showed its degradation activity within 45 days. The surface analysis of the degraded films helps us to identify the degradation effects by bacteria. Hence the use of biological methods to degrade the synthetic polymers can increase the speed of degradation of the synthetic polymers without the production of toxins. The PU degrading bacteria are present in soil so that we can conclude that the nature has solutions for almost all the problems. Hence from this study we can identify that soil microbes can be used for the bioremediation of plastic wastes as well.

\section{ACKNOWLEDGMENTS}

We acknowledge the Department of Biotechnology, Karunya Institute of Technology and Sciences, Coimbatore, India for facilitating the conduct of this article.

\section{CONFLICT OF INTEREST}

The authors declare that there is no conflict of interest. 


\section{AUTHORS' CONTRIBUTION}

All authors listed have made a substantial, direct and intellectual contribution to the work, and approved it for publication.

\section{FUNDING}

None.

\section{DATA AVAILABILITY}

All datasets generated or analysed during this study are included in the manuscript.

\section{ETHICS STATEMENT}

Not applicable.

\section{REFERENCES}

1. Akindoyo J, Beg M, Ghazali S, Islam M, Jeyaratnam $\mathrm{N}$, Yuvaraj A. Polyurethane types, synthesis dand applications - a review. RSC Adv. 2016;6(115):114453114482. doi: 10.1039/c6ra14525f

2. Mahajan N, Gupta P. New insights into the microbial degradation of polyurethanes. RSC Adv. 2015;5(52):41839-41854. doi: 10.1039/c5ra04589d

3. Osman M, Satti S, Luqman A, Hasan F, Shah Z, Shah A. Degradation of Polyester Polyurethane by Aspergillus sp. Strain S45 Isolated from Soil. J Polym Environ.
2017;26(1):301-310. doi: 10.1007/s10924-017-0954-0

4. Gunawan N, Tessman M, Schreiman A, et al. Rapid biodegradation of renewable polyurethane foams with identification of associated microorganisms and decomposition products. Bioresour Technol Rep. 2020;11:100513. doi: 10.1016/j.biteb.2020.100513

5. Kemona A, Piotrowska M. Polyurethane Recycling and Disposal: Methods and Prospects. Polymers. 2020;12(8):1752. doi: 10.3390/polym12081752

6. Shah A, Hasan F, Akhter J, Hameed A, Ahmed S. Degradation of polyurethane by novel bacterial consortium isolated from soil. Ann Microbiol. 2008;58(3):381-386. doi: 10.1007/bf03175532

7. Edgar R. Muscle : multiple sequence alignment with high accuracy and high throughput. Nucleic Acids Res. 2004;32(5):1792-1797. doi: 10.1093/nar/gkh340

8 Talavera G, Castresana J. Improvement of Phylogenies after Removing Divergent and Ambiguously Aligned Blocks from Protein Sequence Alignments. Syst Biol. 2007;56(4):564-577. doi: 10.1080/10635150701472164

9. Dereeper A, Guignon V, Blanc $G$ et al. Phylogeny.fr: robust phylogenetic analysis for the non-specialist. Nucleic_Acids Res. 2008;36(issueSuppl_2):W465-W469. doi: 10.1093/nar/gkn180

10. Gautam R, Bassi AS, Yanful EK. Candida rugosa lipasecatalyzed polyurethane degradation in aqueous medium. Biotechnol. Lett. 2007;29:1081-1086. doi: 10.1007/s10529-007-9354-1 